\title{
Research on the Key Technologies of Transmitter for LED Lighting Communication System
}

\author{
Chao Yang \\ Department of Electronic and Communication Engineering, North China Electric Power Uni- \\ versity, \\ Baoding 071000, China \\ yang199604@qq.com
}

Keywords: Visible light communication, white LED, modulation driving circuit,OFDM

\begin{abstract}
This paper mainly studies the LED illumination of visible light communication system, the key technology on launch. Through the analysis of the basic characteristics of white LED, it respectively designed for analog and digital modulation communication system to the launch of the LED driver circuit. At the same time it solved the following the design requirements:(1)By outputting the stability of the light power as large as possible broaden the coverage system; (2) Making use of the Matlab simulated indoor lighting, receiving the simulation diagram of power and light intensity. Through changing parameters for many times get the best lighting layout. Use OFDM coding technology to reduce the code rate, increased the signal pulse cycle, weakening the multiparty propagation caused by the influence of the ISI, also add protection interval between OFDM signal, further weakening the effect of ISI. At all, the Microcontrollers are used for data transmission test. According to the characteristics of Microcontrollers' serial output data, it set up the launch driver circuit, achieved the desired goal and completed the data transmission very well.
\end{abstract}

\section{Introduction}

White light communication technology is called Visible Light Communication, its principle is to transmit information will need modulation on the drive current of LED lamps and lanterns to shine the LED lamps and lanterns with high frequency .Although the human eye can't see the flash, but through the photoelectric detector can detect the high frequency flicker to carry the communication of information. Its basic structure as shown in the Figure 1 below.

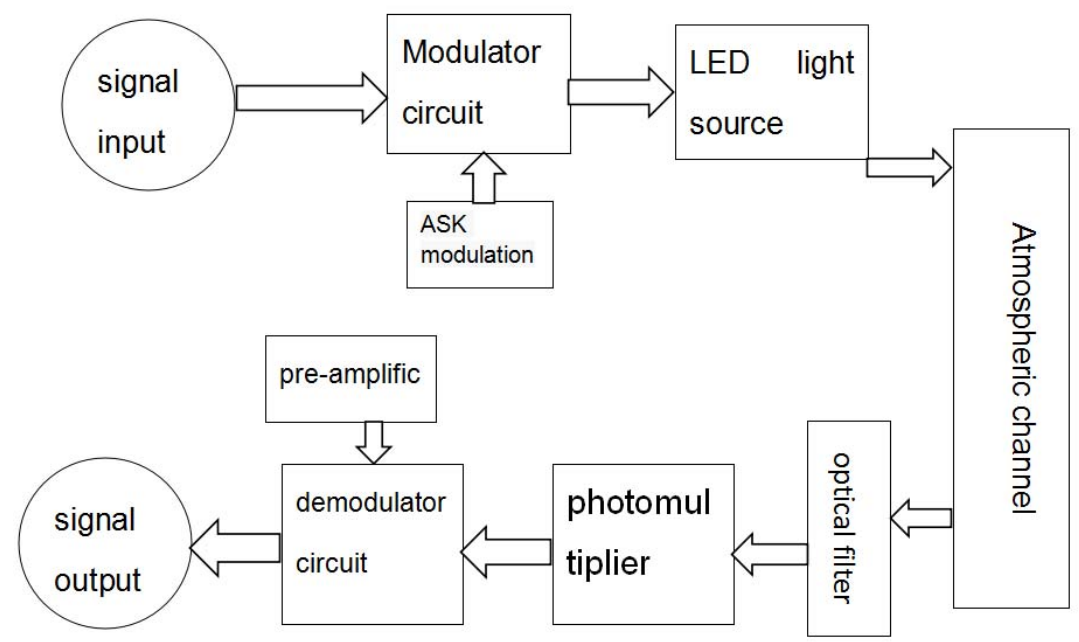

Fig. 1 The principle diagram of the visible light communication

Visible Light Communication technology based on white LED combines the advantages of both optical communications and wireless communication, white LED devices at the same time is the next generation of solid lighting source, the development trend of green is a kind of high-speed flexible, green environmental protection new communication technology, meanwhile it has applica- 
tion foreground huge concluding on many advantages: which optical wavelengths it uses is belong to the white Spaces, broaden the spectrum of broadband communications, and high safety, the secrecy good, coupled with the driving circuit of can communication circuit and LED lamps and lanterns perfect integration.

\section{The research content and papers composition}

\subsection{Visible Light communication}

In this paper, the researches on the visible light communication system based on the LED, mainly studies the key technologies on its launch. This article starts with the analysis of the basic characteristics of white LED by studying its luminous principle, spectrum, volt-ampere characteristic and so on, finally we designed a high-speed modulation driving circuit of LED to complete the transmitting terminal of optical communication system. It mainly includes the following aspects: how to design the high power LED driver circuit, make it cannot only meet the demand of lighting, but also can realize modulation communication requirements; how to reasonable layout of the LED light source, make indoor light environment is so good that there is no communication blind area; how to choose the suitable modulation format, to facilitate transport information and low error rate.

\subsection{The basic characteristic of the white LED}

\subsubsection{White LED light-emitting principle}

LED core part is composed of P-type and N-type semiconductor semiconductor chip, in the transition between the P-type and N-type semiconductor semiconductor layer, is that we often say $\mathrm{P}-\mathrm{N}$ section. When we give a forward voltage of the P-N quarter, changing the dynamic balance of $\mathrm{P}-\mathrm{N}$ section, the injection of minority carrier with majority carrier compound, which will release excess energy in the form of light.

2.2.2Volt-ampere characteristics

Volt-ampere characteristic parameters include forward current, the forward voltage, reverse current, reverse voltage etc. LED lights in only driven by current, voltage to work properly.It is shown below.

\subsubsection{Modulation characteristic}

The response time is short, white LED, as a general rule, be nanoseconds. Therefore, white LED the modulation frequency is very high, we can use it as indoor lighting light source of communication system, its response time of the LED light is extinguishing time, mainly depends on the carrier lifetime, the junction capacitance and impedance circuit.

2.2.4Spectral characteristics

A single LED chip light spectrum depends on the structure of $\mathrm{P}-\mathrm{N}$ junction of band structure of semiconductor materials. White LED is a coherent light source, when the device is working at higher temperatures, the spectral curve will then moves to the right, therefore we need to pay attention to the heat dissipation problem. White LED light is not a single color, that is, not only has a peak wavelength, with multiple peaks, but there is a dominant wavelength.

Through the above introduction to white LED, it is suitable for the visible light communication system.some of the features of induction and the Table1 below: 
Table 1 The LED performance summary

\begin{tabular}{|c|c|}
\hline Characteristics & Range \\
\hline $\begin{array}{l}\text { Modulation } \\
\text { frequency }\end{array}$ & Scores of $\mathrm{KHz}$ to hundreds of $\mathrm{MHz}$ \\
\hline $\begin{array}{l}\text { Channel } \\
\text { rate }\end{array}$ & Theoretical data for a few hundred $\mathrm{Mb} / \mathrm{s}$ \\
\hline $\begin{array}{l}\text { Operating } \\
\text { wavelength }\end{array}$ & $380 \mathrm{~nm}-780 \mathrm{~nm}$ \\
\hline $\begin{array}{l}\text { width } \\
\text { weectum }\end{array}$ & $25 \mathrm{~nm}-100 \mathrm{~nm}$ \\
\hline $\begin{array}{c}\text { Response } \\
\text { time }\end{array}$ & Nanosecond \\
\hline $\begin{array}{l}\text { Transmitted } \\
\text { power } \\
\text { Circuit }\end{array}$ & $\begin{array}{l}\text { White LED launch big power, power limits are generally not } \\
\text { required }\end{array}$ \\
\hline layout & Both lighting information purposes, simplify the circuit layout \\
\hline
\end{tabular}

\section{The Key Technology Research}

\subsection{The modulation of white LED driver circuit design}

White LED driver circuit designed to improve the visible light communication system based on white LED is very important for the extinction ratio and modulation frequency.By optimizing the modulation driving circuit, we can maximize the use of the existing white LED has been commercialized,to realize high speed data transmission.In optical communication channel,we often use Intensity modulation--Direct detection. The basic principle of it is light intensity change with input current signal, the signal waveform is not negative.

3.1.1Analog modulation circuit analysis of white LED

In analog communication has two requirements: first is to provide the appropriate static working point and enough drive current, secondly, the replication of white LED light power output and phase change according to the input signal, nonlinear distortion is small. 


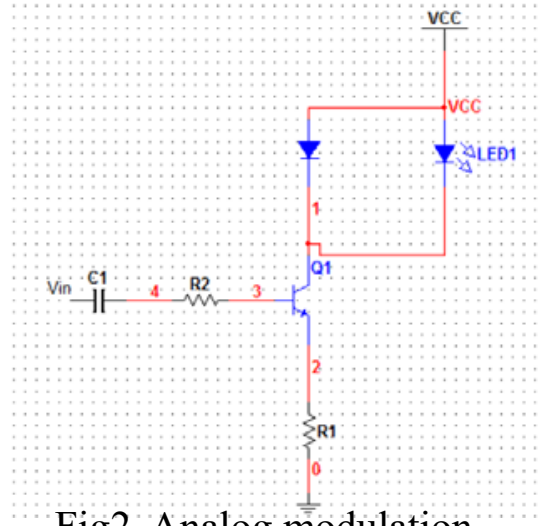

Fig2. Analog modulation

As shown for a total of emitter mutual impedance white LED simulation driving circuit principle diagram. The circuit to enter the base of the electrical signal is transformed into the change of the collector current.Adjust the base bias current to make the circuit work in class A, collector current in static when the LED bar is the white LED the bias current. By changing the base resistance of the resistance to the LED static working time to the appropriate section stress intensity. Circuit using the diode to the single connectivity in order to improve the LED linear.

\subsubsection{Digital modulation circuit}

When white LED light source is used in digital optical communication system, the driving circuit should be able to provide the work within the scope of the "open" and "off" current, only need to add a small amount of positive bias current. The response speed of the white LED to the rapid charge and discharge determines its modulation bandwidth.

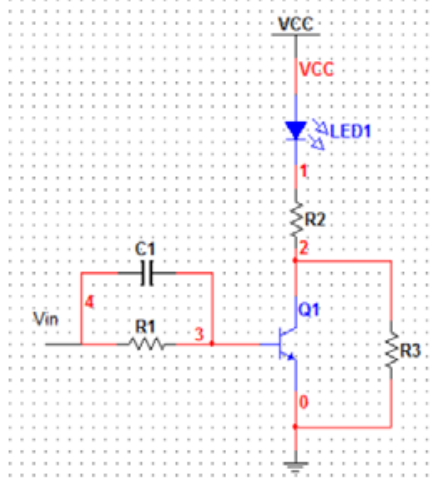

Fig3. Digital modulation

Fig3 is common emitter drive circuit, through the white LED current with VCC and R2 decided to adjust the R2 can make the white LED working current changes, R3 can be provided to the white LED a small amount of bias, in order to improve the switching speed, $\mathrm{C} 1$ to accelerate capacitance, is also in order to improve the switching speed.

\subsubsection{Single chip microcomputer to transmit data experiment}

We use single chip microcomputer for data transmission test microcomputer serial output data, we set up the launch of the following circuit.

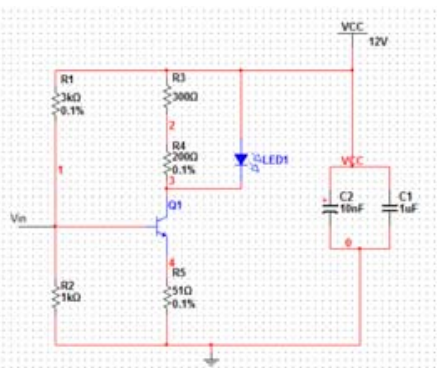

As shown in the Fig4,resistor R3 and R4 and white LED have the effect of shunt which avoid excessive current through the LED, can also control transistor with resistor R1, R2 and R5 to work in such A state. Experiments through the potentiometer to adjust the resistance of the right, then choose the appropriate R3 and R4, capacitor $\mathrm{C} 2, \mathrm{C} 3$ have the effect of filter out high frequency and low frequency noise signal.

Fig4. Test circuit

Actual connection as shown in the figure below, we can use it to achieve the expected goals, complete the data transmission, send data by a single chip microcomputer, another MCU receives the data, and the data on the LCD1602 shows it received. We realized the data transmission of 70 $\mathrm{cm}$.

For improvement we have also set up a voltage stabilizing circuit, so that we can make the output stability, LED output is more stable.

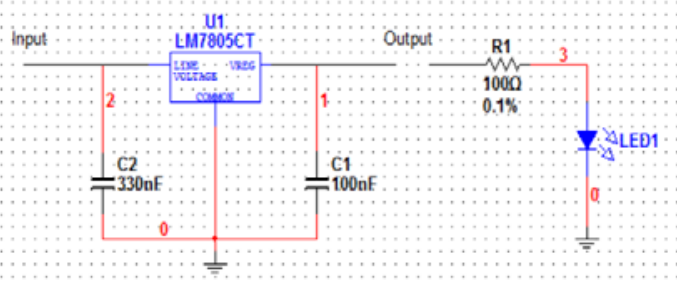

In the Fig5,use of a resister to regulate current through the LED is required in fixed voltage regulators.As shown in the formulal below.

$$
I_{f}=\frac{\left(V_{\text {out }}-V_{f}\right)}{R}
$$

Fig5. Voltage regulator 


\subsection{The Light Source Layout}

To meet the demand of basic lighting, in system design we should first consider the distribution of the indoor light. To attain the optimal communication effect, we must according to the size of the room and indoor facilities different rational layout to make the room within the intensity distribution is roughly same, as well as trying to avoid blind area (less than the light areas).

Keeped out of people, equipment, etc, it will be formed on the surface of the receiver "shadow", affecting the communication performance. For lighting, indoor the more installation of lighting the more you can reduce the "shadow" effect,but a number of different optical path makes the more serious the ISI. While to meet indoor illumination standard,we also want to consider the effect of ISI, it is important to arrange the layout of the LED light source array, we can use MATLAB to simulation, setting up the corresponding parameter, and then obtained the received power and light intensity of the simulation diagram, through changing parameters for many times, to get the best lighting layout.

\subsection{Channel coding technology}

We adopted On-Off Keying(OOK direct intensity modulation)in the experiment.It is based on the single polarity is not zero code sequence to control the opening and closing of the sine carrier, namely in the visible light communication with "0", "1" to control the LED light and dark.Its implementation is simpler, we can use a transistor to control implementation, but it is limited in transmission rate and poor anti-interference.

We can use OFDM code for improvement. The main idea of the OFDM technology: in frequency domain will be given channel into multiple orthogonal Sub-channels, using sub-carrier modulation on each sub channel, and each subcarrier parallel transmission. Make each relatively flat sub-channels, and on each channel is a narrow band transmission, less than the coherence bandwidth of channel signal bandwidth. Therefore, this method can greatly eliminate the ISI.In the visible light communication OFDM system, must first to OFDM signal source signal encoding, then run offset of LED light source modulation. Because it will be in a serial of high speed data in parallel to the multiple orthogonal sub-carrier modulation, reduce the code rate, increase the signal pulse cycle, weaken the multiparty propagation caused by the impact of the ISI . On the other hand, it can add protection interval between OFDM signal, which further weaken the effect of ISI.

\section{Conclusion}

Visible light communication is an emerging wireless optical communication technology, has become a new growth point of optical wireless communication field, with the depth of research, its application will become more and more widely.

Visible light communication have not occupy spectrum, high transmission power, no electromagnetic interference, the advantages of saving energy, has great prospects for development, but to really implement the indoor high speed wireless data communication, and there are many places can be improved, in basically has the following aspects:1.how to improve the production process, the LED in the higher transmit power in order to meet the communication distance at the same time, not so bright.2. how to choose more appropriate coding technology to reduce the ISI. 3 how to achieve a higher rate of transmission by optimizing circuit.

\section{Reference}

[1]Song Jian Bao. LED Visible Light Communication System Design and Performance Optimization Based on Ethernet [J]. Applied Mechanics and Materials, 2013, 2491(336):

[2]Huanhuan Zheng,Jian Chen,Changyuan Yu,Mohan Gurusamy. Inverse Design of LED Arrangement for Visible Light Communication Systems[J]. Optics Communications,2016,:.

[3]Song Jian Bao. LED Visible Light Communication System Design and Performance Optimization Based on Ethernet[J]. Applied Mechanics and Materials,2013,2491(336):. 
[4]Huanhuan Zheng,Jian Chen,Changyuan Yu,Mohan Gurusamy. Inverse Design of LED Arrangement for Visible Light Communication Systems[J]. Optics Communications,2016,:.

[5] Information on: https://ledlight.osram-os.com/knowledge/led-fundamentals/ 